\title{
Bright light transmits through the brain: Measurement of photon emissions and frequency-dependent modulation of spectral electroencephalographic power*
}

\author{
Michael A. Persinger ${ }^{1,2,3 \#}$, Blake T. Dotta ${ }^{1,2}$, Kevin S. Saroka ${ }^{1,3}$ \\ ${ }^{1}$ Behavioural Neuroscience Program, Laurentian University, Sudbury, Canada \\ ${ }^{2}$ Biomolecular Sciences Program, Laurentian University, Sudbury, Canada \\ ${ }^{3}$ Human Studies Program, Laurentian University, Sudbury, Canada \\ Email: ${ }^{*}$ mpersinger@,laurentian.ca
}

Received 27 October 2012; revised 28 November 2012; accepted 19 December 2012

\begin{abstract}
Photons are emitted during brain activity and when applied externally alter its functional connectivity during the resting state. In the present study we applied constant or time varying light $(\sim 10,000$ lux $)$ stimuli to one side of the skull and measured by photomultiplier tubes the photon density emitted from the opposite side of the skull along its two horizontal planes. Global quantitative electroencephalographic activity (QEEG) was recorded simultaneously. Reliable increases of $\sim 2.5 \times 10^{-11} \mathrm{~W} \cdot \mathrm{m}^{-2}$ during either constant or specific flash frequencies between 3 and $7 \mathrm{~Hz}$ as well as enhanced QEEG power in the theta and low beta range were measured. According to source localization by Low Resolution Electromagnetic Tomography (LORETA) the right parahippocampal region was particularly enhanced. Calculations employing known quantitative values for permeability and permittivity of brain tissue were consistent with this frequency band. Estimated concentrations of protons from hydronium ions indicated a Grotthuss chain-like process for moving photon energy through the cerebral medium may have mediated the distance-dependent latency. The results suggest that external light is transmitted through cerebral tissue, can be measured externally, and significantly affects functional connectivity. The findings support the conclusions of Starck et al. (World Journal Neuroscience, 2012).
\end{abstract}

Keywords: Cerebrum; Intracerebral Light Transmission; Photon Density; Quantitative Electroencephalography (QEEG); LORETA (Low Resolution Electromagnetic Tomography)

\footnotetext{
"There are no conflicts of interest.

${ }^{\#}$ Corresponding author.
}

\section{INTRODUCTION}

Within the last three decades there have been multiple reports of the emissions of Ultraweak Photon Emissions (UPE) from brain tissue [1]. In addition to the approximately $10^{6}$ photons $\cdot \mathrm{m}^{-2} \cdot \mathrm{s}^{-1}$ that are measurable from surfaces of living organisms [2], slices of hippocampal tissue emit photon densities of $\sim 10^{-12} \mathrm{~W} \cdot \mathrm{m}^{-2}$ that are coupled to theta-activity [3]. Recently Bokkon and his colleagues [4] reported that retinal tissue from rats emits photons in response to exogenous light stimulation, an observation compatible with several theoretical and quantitative solutions [5].

We have been examining the intricacies of photon emissions from the human brain, as measured by photomultiplier tubes (PMT), during protocols for imaging vs not imaging white light while subjects sat in complete darkness $[6,7]$. We then asked the question if very bright light (about 10,000 lux) applied tightly against the skull could traverse cerebral space and be discerned by PMTs and affect the power of specific bands of quantitative electroencephalographic (QEEG) activity on the opposite side of the skull. Here we present evidence that reliable changes for both measures can be discerned.

That photons can traverse the skull and influence biochemical and biophysical functions within brain space has been known or suspected by many previous researchers [8]. The pineal organ is responsive to external light sources even when the neural input is removed. Although frequency-dependent changes in QEEG activity in response to external light flash intervals are so reliable they are routinely employed for neurological diagnosis of some types of epilepsy, the effects of light flashed against the scalp for a person blinded folded sitting in the dark has not been systematically investigated. There are also the more recent discoveries of light sensitive molecules distributed throughout the human brain [9] which have been coupled to release of gamma-aminobutyric acid [10] and mobilization of intracellular calcium [11]. For the pre- 
sent experiments we reasoned that if the effect was biophysically robust a reliable effect should be evident by only triplicate replication ( 3 subjects) which is the normal procedure to assess most biochemical reactions.

\section{MATERIALS AND METHODS}

\subsection{Experiment 1: Constant Light Transmission}

In Experiment 1, we examined the density of photons that emerged on the contralateral skull when light was applied. There were three subjects that were measured over a two month period. Each subject sat in a comfortable chair within a completely darkened room. In separate experiments (different days) for each subject a commercial LED flashlight (4 cm wide) was applied either: 1) directly below the occipital notch so that the linear path of the light emerged through the forehead, or 2) along the left temporal area so the light would emerge from the right temporal region.

The flashlight was modified so that it could be turned on or off in a second room where the PMT was maintained. The flash light was also wrapped with dense black terry cloth material around the subject's head to insure no stray light occurred. This was verified by the absence of any immediate change in PMT reading when the light was on or off.

A RCA electron tube (no filters) within a BCA IP21 housing (the PMT) was placed $15 \mathrm{~cm}$ on the side opposite to the flashlight. The PMT output was connected to a millivolt meter and to a SRI Pacific Instruments photometer. The output from the 0 to 100 unit scale was recorded 3 times per second (maximum limit of the software) to an IBM ThinkPad laptop (Windows 95). Based upon three different methods of calibration, an increase of 1 unit was equivalent to $5 \times 10^{-12} \mathrm{~W} \cdot \mathrm{m}^{-2}$ [12]. We selected $30 \mathrm{~s}$ for the trial duration based upon pilot experiments.

For each subject and each experiment there were four sequential sets of $30 \mathrm{~s}$ intervals for light off and light on conditions. The subjects were either told or not told when the light was activated (a procedure that did not alter the results) and were instructed to simply relax. To insure there was no light scatter through the dense material the light was activated against a $2 \mathrm{~cm}$ thick pine board and wrapped in the same manner. The PMT was placed $15 \mathrm{~cm}$ on the other side of the board. Comparison of the 90 measurements when the light was on (30 s) vs off (30 s) indicated no statistically significant difference between the two conditions.

\subsection{Experiment 2: Frequency-Varying Light Transmission: Quantitative EEG (QEEG) Measurements}

In Experiment 2 and 3 different volunteers each sat blind-folded in a high-back, soft arm chair that was housed in a dark industrial acoustic chamber that was also a Faraday cage (a different room than in Experiment 1). Quantitative EEG activity was recorded at $250 \mathrm{~Hz}$ with a Mitsar-201 amplifier equipped with 19-channel sensors. The cap was fit comfortably for each subject whose head rested slightly backward for relaxation. The face of a different (but same type) flashlight was placed against the back of the skull as in Experiment 1. The light was held in place by black thick soft terry cloth towels that also wrapped around the flash light. The frequencies of light flashes were controlled by a Heath-Schlumberger functional generator. Both the function generator and the laptop recording the EEG output from the cap were housed externally to the chamber.

The following light flash frequencies were employed: all integers from 1 to $10 \mathrm{~Hz}$. In some trials the $0.5 \mathrm{~Hz}$ intermediate frequencies, e.g., $7.0 \mathrm{~Hz}, 7.5 \mathrm{~Hz}, 8.0 \mathrm{~Hz}$ between each integer were inserted. The frequencies were presented in quasi random order between minimum and maximum and were in different orders for each subject. Each flash frequency was presented for $30 \mathrm{~s}$ followed by a $30 \mathrm{~s}$ period of no flash. The subjects reported they only experienced complete darkness and no sounds of any kind.

According to our usual protocol $[13,14]$, spectral analyses and power in $\mu \mathrm{V}^{2} \cdot \mathrm{Hz}^{-1}$ were integrated over traditional frequency bands by employing Matlab software for delta (1 to $3.9 \mathrm{~Hz}$ ), theta (4 to $7.9 \mathrm{~Hz}$ ), low alpha $(8.0$ to $10.5 \mathrm{~Hz})$, high alpha (10.6 to $13 \mathrm{~Hz})$, low beta (13.1 to $20 \mathrm{~Hz}$ ) high beta $(21$ to $35 \mathrm{~Hz}$ ) and gamma (35.1 to 50 $\mathrm{Hz}$ ). The data from the digitized EEG were plotted as a function of frequency for each subject. $30 \mathrm{~s}$ segments corresponding to the presentation of the light frequencies were imported into LORETA (LOw Resolution Electromagnetic TomogrAphy) for source localization [15]. On the bases of the preliminary most significant results for systematic frequency-dependent changes over the various lobes for the three subjects, the LORETA to ROI (region of interest) function was employed to calculate activation within classic frequency bands. The area selected was the right parahippocampal gyrus because of its multimodal input and output to cerebral cortical areas. These data were subsequently imported into SPSS software for further analyses. To accommodate individual differences and drift fluctuations from sitting in a chamber, the QEEG changes for any light-frequency flash interval from the immediately previous no-flash interval (relative changes in power) were calculated.

\subsection{Experiment 3: Light Emission from the Brain During Frequency-Varying Light}

In Experiment 3, 3 different volunteers sat within the same chamber as in Experiment 2 and were exposed to the 
same procedure. However a digital photometer Model DM009C (Senstech Ltd.) was placed $15 \mathrm{~cm}$ directly in front of the forehead. The difference between this PMT and the one employed in Experiment 1 was that it measured numbers of photons per sample rather than $\mathrm{W} \cdot \mathrm{m}^{-2}$. Sampling rate was $50 / \mathrm{s}(20 \mathrm{msec}$ intervals) for the entire duration of exposure for each subject. The numbers of photons per sample for each of the light flash frequencies (repeated twice for every subject) were transported to SPSS-PC software for analyses.

\section{RESULTS}

\subsection{Experiment 1: Constant Light Transmission}

The typical result for each subject for the repeated off-on (30 s each) design for measuring photon changes through cerebral space when light was presented to the left temporal skull and photon density was measured on the opposite (right side) of the skull is shown in Figure 1. The values refer to the actual photometer units during the successive $30 \mathrm{~s}$ intervals when the light was applied (on) or not applied (off). The vertical bars refer to the SEMS for the 90 measurements $(3 / \mathrm{s})$ within each $30 \mathrm{~s}$ interval. Compared to the off condition, the on conditions were associated with a net increase of between 0.5 and 1 unit or $2.5 \times 10^{-11} \mathrm{~W} \cdot \mathrm{m}^{-2}$ to $5 \times 10^{-11} \mathrm{~W} \cdot \mathrm{m}^{-2}$. The effects were similar for each of the three subjects.

The latencies for the point-to-point $(0.33 \mathrm{~s})$ increases in photon density for the 3 samples per sec measurements when the light was activated along the major axis (caudal to frontal skull) or the minor axis (left to right temporal lobe) are shown in Figure 2. In order to compare the two measurements directly z-scores for the photon units were computed for the $1 \mathrm{~s}$ before to $5 \mathrm{~s}$ after the light activation for the various trials. The latency for photon emission to increase during lateral (side to side) application was $0.66 \mathrm{~s}$ while the caudal-rostral latency was about $1.66 \mathrm{~s}$. The absolute increase in photon emission ranged between 0.5 to 0.6 units or about $2.5 \times 10^{-11}$

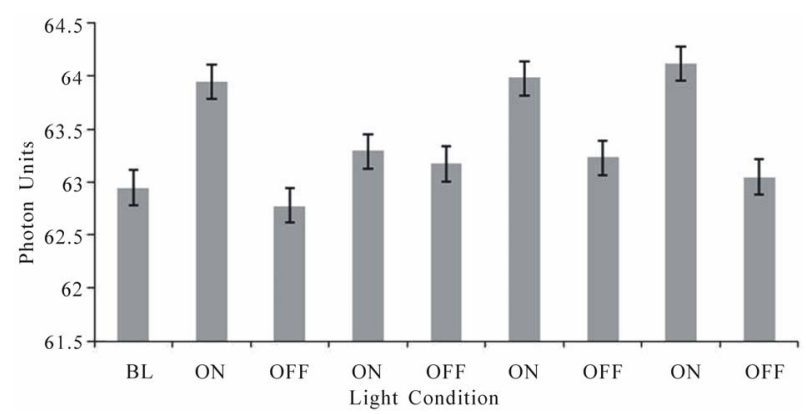

Figure 1. Photon units $\left(1\right.$ unit $\left.=5 \times 10^{-11} \mathrm{~W} \cdot \mathrm{m}^{-2}\right)$ on the right side of the head during periods when a light source placed tightly over the left side of the head was on or off. Each interval wasa $30 \mathrm{sec}$. BL is baseline. Vertical bars indicate standard errors of the mean for 90 successive measurements.
$\mathrm{W} \cdot \mathrm{m}^{-2}$

\subsection{Experiment 2: Frequency-Varying Light Transmission: Quantitative EEG (QEEG) Measurements}

Seven separate MANOVAs with two within subject factors (frequency of light and left-right hemisphere) were performed for each frequency band. There were only two statistically significant changes as a function of light flash frequency for the source-localization data. These occurred within the right paraphippocampal gyrus. The first was a statistically significant frequency effect $(\mathrm{F}(7,28)=$ $3.14, p<0.05$; eta $^{2}=0.44$ ). Post hoc analysis indicated the major source of the effect, as shown in Figure 3, was the increase in power within the theta range within the right parahippocampal gyrus during the presence of the 7 $\mathrm{Hz}(6.5$ to $7.5 \mathrm{~Hz})$ light flashes.

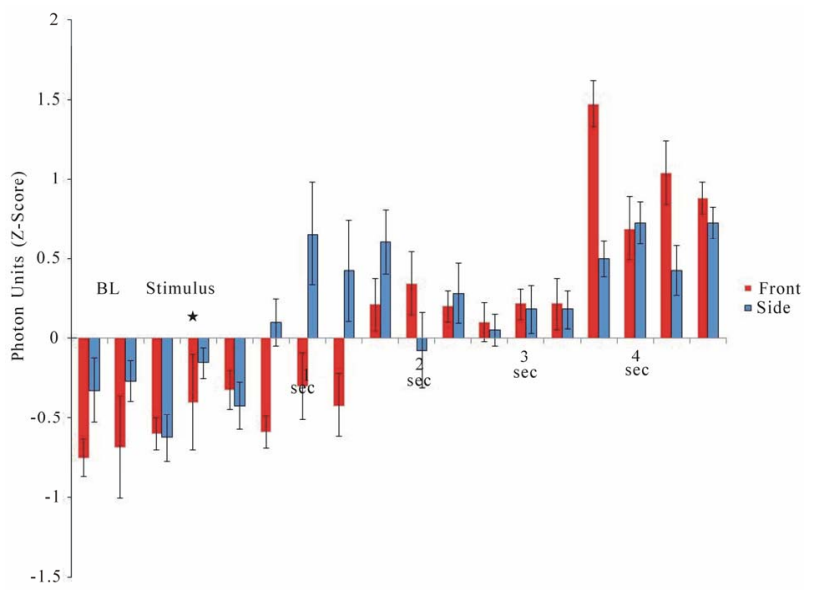

Figure 2. Latency of response from the onset of the light to the emergence of photon emission from the right side (from left side stimulation) or from the front of the skull (from application of light to the caudal scalp). Vertical bars are SEMs. BL = baseline; $*$ indicates application of light stimulus.

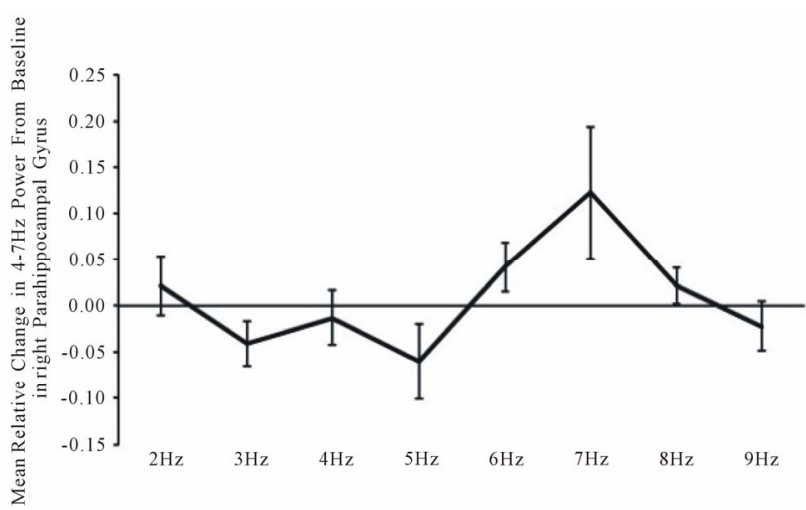

Figure 3. Relative change from baseline in power within the theta $(4-7 \mathrm{~Hz})$ band within the right parahippocampal gyrus as a function of the frequency of light flash frequency delivered against the back of the head. 
Within the low beta $(13-20 \mathrm{~Hz})$ EEG band, MANOVA revealed a statistically significant $(F(7,28)=$ $3.58, p<0.05$; eta $^{2}=0.47$ ) two-way way interaction between power and light flash frequency within the right parahippocampal region. Post hoc analyses indicated that the major source of this interaction was the increase in power within the low beta band when the 4 to $5 \mathrm{~Hz}$ and 9 $\mathrm{Hz}$ stimulations were applied relative to the $2 \mathrm{~Hz}$ presentations (Figure 4).

\subsection{Experiment 3: Light Emission from the Brain During Frequency-Varying Light}

For all three subjects there was a clear frequency-dependence between the light flash frequency delivered through the caudal scalp and the output of photons from the front of the skull. As can be seen in Figure 5, there were significant increases in the photon emissions from

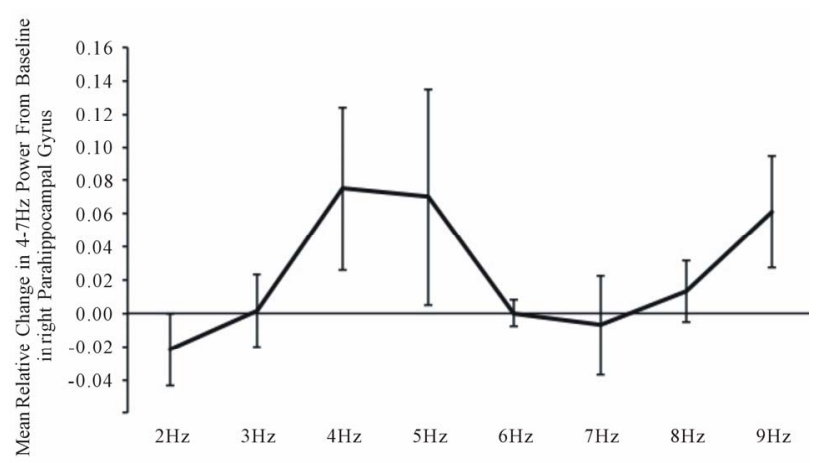

Figure 4. Relative changes in power compared to baseline in the low beta range within the right parahippocampal region as a function of the light flash frequencies.

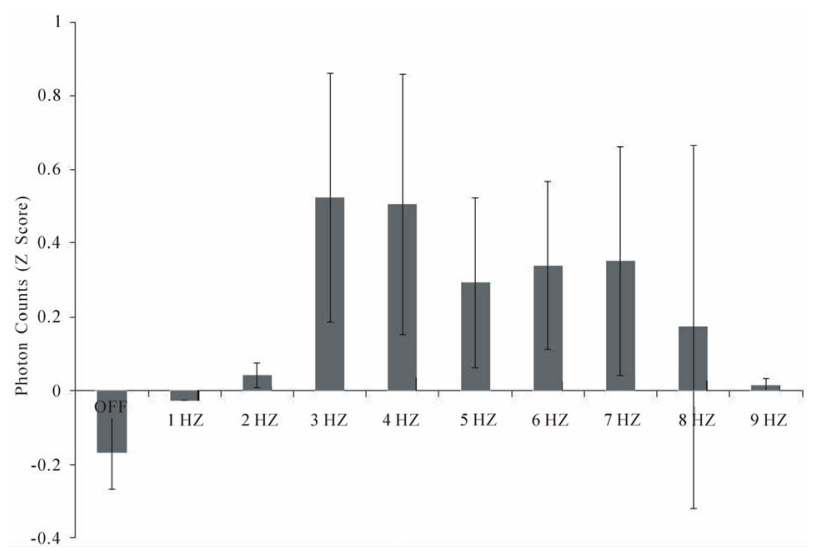

Figure 5. Photon counts $15 \mathrm{~cm}$ from the forehead of subjects blindfolded sitting in the dark during frequencies (1 to $9 \mathrm{~Hz}$ ) of light applied against the back of the skull (and covered completely with black terrycloth). Data are averages of two trials for each subject and 3 subjects. Vertical bars indicate standard deviations. There were 1200 samples for each frequency for each subject. A change of $0.5 \mathrm{z}$ is equivalent to 250 photons $/ 20$ ms sample. the brain when the light flash frequency ranged between 3 and $7 \mathrm{~Hz}$, compared to $1-2 \mathrm{~Hz}$ or $9 \mathrm{~Hz}$ or compared to the no light condition. The maximum deviation (z-score of 0.50 ) is equivalent to an average of 250 photons $/ 20 \mathrm{~ms}$ interval over the $30 \mathrm{~s}$ interval for a given light flash frequency.

The data are means of standardized scores of the within subject z-scores for each subject. The difference in average absolute background photon counts from each subject was equivalent to $2.5 \times 10^{1}$ photons per $25 \mathrm{~ms}$ interval or $1.25 \times 10^{4}$ photons $\cdot \mathrm{s}^{-1}$. Assuming the median wavelength was $555 \mathrm{~nm}$, each photon would be $0.54 \times$ $10^{15} \mathrm{~Hz}(1 / \mathrm{s})$ and when multiplied by Planck's constant $\left(6.62 \times 10^{-34} \mathrm{~J} \cdot \mathrm{s}\right)$ would be $3.6 \times 10^{-19} \mathrm{~J}$ per photon or a total of $4.5 \times 10^{-15} \mathrm{~J} \cdot \mathrm{s}^{-1}$. Given the aperture of the digital PMT was $\pi \times 10^{-4} \mathrm{~m}^{2}$, the equivalent power would be $1.4 \times 10^{-11} \mathrm{~W} \cdot \mathrm{m}^{-2}$ which is almost identical to that measured by the analog PMT.

\section{DISCUSSION}

The results of these studies indicated that approximately 10,000 lux of steady white light when applied against the skull for successive presentations of $30 \mathrm{~s}$ on and $30 \mathrm{~s}$ off conditions results in detectable and reversible increases in photon emission from the contralateral side. The measurements were not likely to be artifact for three reasons. First, the time required for the response was much slower than that when light was activated in free space. Second, the latency along the rostral-caudal axis $(\sim 1.3 \mathrm{~s})$ vs the lateral-lateral (side-to-side) axis $(\sim 0.7 \mathrm{~s})$ was proportional to the actual linear distance for the subjects' skulls. Third, when the same procedure was completed with a 2 $\mathrm{cm}$ thick pine board, there were no changes in photon detection.

The discernable latency (about $1 \mathrm{~s}$ ) to traverse cerebral space would require a substantial slowing of the velocity of the photons. If one assumes a Grotthuss-like chain reaction, then a species of matter with a specific density and life-time could accommodate this latency. One candidate is the hydronium ion whose concentration in physiological systems is about $40 \mathrm{nM}$ and displays a life time is about $10^{-12} \mathrm{~s}$ [16]. If one assumes the classic Grotthuss chain, then the time for a quantum of energy to traverse a volume through the mobile $\mathrm{H}^{+}$(proton) can be estimated.

The mobility of a proton in water is $3.6 \times 10^{-3}$ $\mathrm{cm}^{2} \cdot \mathrm{V}^{-1} \cdot \mathrm{s}^{-1}$. Assuming a potential difference of $10^{-1} \mathrm{~V}$ across the rostral-caudal boundaries, in $1 \mathrm{~s}$ the mobility would be $3.6 \times 10^{-4} \mathrm{~cm}^{2}$ and through $15 \mathrm{~cm}$ of linear brain space would involve $5.4 \times 10^{-3} \mathrm{cc}$ or $5.4 \times 10^{-6} \mathrm{~L}$. This value multiplied by $\left(40 \times 10^{-9} \mathrm{M}\left(\mathrm{H}^{+}\right) / \mathrm{L}\right) \times(6.023 \times$ $10^{23}$ molecules/Mole) $\times 10^{-12} \mathrm{~s} /$ hydronium molecule) results in a traversal latency of $\sim 0.13 \mathrm{~s}$ for the population. If one assumes only about $25 \%$ of cerebral space is ex- 
tracellular fluid, then the time would 4 times slower or about $0.5 \mathrm{~s}$, which is within the range of measurement of our observations.

When the light stimuli was flashed (similar light source) within the major power spectrum of the electroencephalographic range (1 to $10 \mathrm{~Hz}$ ), the photon emissions from the contralateral side of the skull as measured by a different (digital) PMT at $15 \mathrm{~cm}$ were comparable in power density to that measured with the analog equipment. Not all frequencies displayed the optimal transparency. Maximum transmission occurred between 3 and $7 \mathrm{~Hz}$. The absence of an effect for either 1 or $2 \mathrm{~Hz}$ or 8 or $9 \mathrm{~Hz}$ suggests that the enhanced was relatively precise and not an artifact of the time-variation.

A similar enhancement of electroencephalographic power occurred within the right parahippocampal region of the subjects. We selected this region rather than whole brain power because of its conspicuous enhancement when the light was activated through the brain as discerned by LORETA. There was clear convergence between the flash frequency of $7 \mathrm{~Hz}$ and increased power within the theta $(4-7 \mathrm{~Hz}$ ) band (Figure 3) within this region in particular although it was evident globally. This pattern is similar to the canonical correlations of brain electric spectral states during spontaneous conscious covert cognition measured by Lehmann et al. [17]. They found a strong concordance $(r=0.68)$ with a power peak between $4-7 \mathrm{~Hz}$ over the medial right hemisphere during passive cognition. The medial cortices, particularly the parietal lobe have significant connections with the pa- rahippocampal regions which we observed to be activated during intracerebral transmission of external light.

Assuming a permeability (inductance $\cdot \mathrm{m}^{-1}$ ) at $1 \mathrm{kHz}$ (functional duration of an action potential, i.e. $10^{-3} \mathrm{~s}$ ) is about $10^{-2}$ Henrys (calculated by dimensional analysis from conductivity in $\mathrm{S} \cdot \mathrm{m}^{-1}$ ) and a permittivity for grey matter is $2 \times 10^{-1} \mathrm{~F} \cdot \mathrm{m}^{-1}$, the formula $\mathrm{f}=\left(2 \pi \times(\mathrm{LC})^{-1 / 2}\right)^{-1}$ results in a resonance frequency of about $7 \mathrm{~Hz}$ [18]. This congruence between the physical properties of cerebral tissue and the resonant frequency, the narrow range of the light flash effect on power enhancement upon quantitative electroencephalographic changes, and the optimal transparency for frequency-varying bursts of photons indicate that the applied light may directly influence intracerebral space and the neurons within it through resonance-related mechanisms.

The validity of the observations is supported by the additional increased power within the 13 to $20 \mathrm{~Hz}$ (beta) range that occurred during the $4-5 \mathrm{~Hz}$ light flashes. The discrepancy between the applied light flash frequency and the frequency band in which the power increased indicates one was not an artifact of the other. Again, as shown by the original studies of Lehmann et al. [17] the first canonical EEG variables associated with ideation involve prominent peaks within the $2-6 \mathrm{~Hz}$ and $13-15$ $\mathrm{Hz}$ band.

The results of this study are commensurate with the recent elegant experiments by Starck et al. [19]. While applying LED-generated light through the ear canals, they found a gradual increase in the functional connectivity as measured by BOLD (Blood Oxygen Level Dependent) MRI across the extrastriate visual cortices that included the dorsal and ventral visual "streams". By extrapolating from their graphs, the effect would have been apparent within about $40 \mathrm{~s}$ and according to their observations asymptoted after about six minutes. The area encountering most of the light, because of their method of application, involved the inferior temporal lobe, anterior cerebellum and pons.

The activation of the parahippocampal region inferred from our measurements is consistent with excitatory functional pathways. There are direct afferents from visual (Brodmann 20) and auditory (Brodmann 22) areas, particularly the caudal third (the posteroventral portion of the angular gyrus) of the inferior parietal lobule (Brodmann 7a), to the paraphippocampal region. It is a component of a mutually interconnected multimodal area that involves projections from areas that include the retrosplenial cortex, superior temporal sulcus, and medial pulvinar. Fibers from the angular gyrus and superior occipital gyrus are also connected with the parahippocampal gyrus [20]. Consequently it could be responsive to light-activation of similar pathways.

Input from the entire cortical mantle converges upon layers II and III of the entorhinal cortices, a major component of the parahippocampal gyrus. Axons from the entorhinal cortices are the origins of the perforant pathway which is the major input to the hippocampus where synaptic interactions occur within the molecular layer of the dentate gyrus and the lacunosum-moleculare layer of CA3. Clusters of stellate cells in layer II of the entorhinal cortices possess a conspicuous intrinsic rhythmic, subthreshold oscillation with a peak around $8 \mathrm{~Hz}$ [21]. This periodicity is remarkably convergent with the interval of maximum light transparency and enhancement of EEG power measured in our studies.

Starck et al. [19] applied intensities of 7 lumen from which lux can be discerned by dividing by $4 \pi r^{2}$. The average diameter of the auditory canal is $3.7 \mathrm{~mm}$ [22]. This would be equivalent to about $1.4 \times 10^{5} \mathrm{lux}$ (comparable to the range of direct sunlight). Assuming 1 lux equals about $1.5 \times 10^{-3} \mathrm{~W} \cdot \mathrm{m}^{-2}$ within the green wavelength, the energy density of the photons from their light emissions would have been $\sim 2.1 \times 10^{2} \mathrm{~W} \cdot \mathrm{m}^{-2}$ and when accommodating the cross-sectional area of the auditory canal would be $\sim 8 \times 10^{-4} \mathrm{~J} \cdot \mathrm{s}^{-1}$. Given the approximately $10^{-12} \mathrm{~J}$ involved with the metabolism of glucose per average neuron and glial cell [23], this would indicate that 
about $8 \times 10^{8}$ cells would have been activated potentially by light energy equivalence. The significant increase in functional connectivity within the ventral and dorsal stream neurons involved $5.2 \mathrm{cc}$. Assuming $10 \mu \mathrm{m}$ widths per cell $\left(5.2 \times 10^{-10} \mathrm{cc}\right)$ and the occupation of about $10 \%$ of the space by somas this volume would involve about $10^{9}$ neurons. Thus there was convergence between the volume affected, the estimated number of neurons within this volume, and the energetic contribution from the applied photon source.

The observation that the group exposed to light showed an elevation of the spectrum at frequencies below 0.035 $\mathrm{Hz}$, that is longer than periods of $29 \mathrm{~s}$, suggests that there are intrinsic variations within neuronal populations that respond to the passage of intracerebal light. We had selected the $30 \mathrm{~s}$ on, $30 \mathrm{~s}$ off paradigm in our studies based upon our pilot data that indicated these durations were required to obtain visually discernable real time changes in photon emission and QEEG power. Whereas the latter required about $20 \mathrm{~s}$ to $30 \mathrm{~s}$ to emerge and asymptote, the former required about $1 \mathrm{~s}$ to be evident but displayed periodic fluctuations within the $30 \mathrm{~s}$ range.

These results support the observations by Starck et al. [19] who employed a different method of measurement. The increased power within the theta and low beta range that we observed during light transmission through brain space would be consistent with enhanced connectivity of cerebral neurons within specific areas. These areas are grossly related to discerning where and what events occur within the environment. If patterns of photons move as slowly through the cerebral medium as our results indicate then the traversal time for the width of a neuronal soma or the average length of an intracortical axon would be within the millisecond range. This congruence could be sufficient to allow direct interaction with information processing within neural circuits [24].

\section{ACKNOWLEDGEMENTS}

We thank Dr. Ed Bosarge, Jr. Chairman, Quantalab LLC for his contributions and Viger M. Persinger for technical comments. Portions of this research were funded by an Ontario Graduate Scholarship

\section{REFERENCES}

[1] Popp, F.A., Li, K.H., Mei, W.P., Galle, M. and Neuohr, R. (1988) Physical aspects of biophotons. Experientia, 44, 567-585. doi:10.1007/BF01953305

[2] Popp, F.A. (1979) Electromagnetic information. Urban and Schwarzberg, New York.

[3] Isojima, Y., Isoshima, T., Nagai, K., Kikuchi, K. and Nakagawa, H. (1995) Ultraweak bioluminescence detected from rat hippocampal slices. NeuroReports, 6, 685-660. doi:10.1097/00001756-199503000-00018

[4] Wang, C., Bokkon, I., Dai, J. and Antal, I. (2011) First experimental demonstration of spontaneous and visible light-induced photon emission from rat eyes with particular emphasis on their roles in discrete noise and retinal phosphenes. Brain Research, 1369, 1-9. doi:10.1016/j.brainres.2010.10.077

[5] Bokkon, I., Salari, V., Tuszynski, J.A. and Antal, I. (2010) Estimated numbers of biophotons involved in the visual perception of a single-object image: Biophoton intensity can be considerably higher inside cells than outside. Journal of Photochemistry and Photobiology B, 100, 160-166. doi:10.1016/j.jphotobiol.2010.06.001

[6] Dotta, B.T., Saroka, K.S. and Persinger, M.A. (2012). Increased photon emission from the head while imaging light in the dark is correlated with changes in electroencephalographic power: Support for Bokkon's biophoton hypothesis. Neuroscience Letters, 513, 151-154. doi:10.1016/j.neulet.2012.02.021

[7] Dotta, B.T., Buckner, C.A., Lafrenie, R.M. and Persinger, M.A. (2011) Photon emissions from human brain and cell culture exposed to distally rotating magnetic fields shared by separate light-stimulated brains and cells. Brain Research, 1388, 77-88. doi:10.1016/j.brainres.2011.03.001

[8] Weaver, D.R. and Reppert, S.M. (1989) Direct in utero perception of light by the mammalian fetus. Developmental Brain Research, 47, 151-155. doi:10.1016/0165-3806(89)90119-3

[9] Taartelin, E.F., Bellingham, J. Hankins, M.W., Foster, R. G. and Lucas, R.J. (2003) Neuropsin (OPN5): A novel opsin identified in mammalian neural tissue. FEBS Letters, 554, 410-416. doi:10.1016/S0014-5793(03)01212-2

[10] Wade, P.D., Taylor, J. and Siekevitz, P. (1988) Mammalian cerebral cortical tissue responds to low-intensity visible light. Proceedings of the National Academy of Sciences of the United States of America, 85, 9322-9326. doi:10.1073/pnas.85.23.9322

[11] Nieto, P.S., Valdez, D.J., Acosta-Rodriquez, V.A. and Guido, M.E. (2011) Expression of novel opsins and intrinsic light responses in the mammalian retinal ganglion cell line RGC-5. Presence of OPN5 in the rat retina. PLoS One, 5, e26147.

[12] Dotta, B.T., Buckner, C.A., Cameron, D., Lafrenie, R.F. and Persinger, M.A. (2011) Biophoton emissions from cell cultures: Biochemical evidence for the plasma membrane as the primary source. General Physiology and Biophysics, 30, 301-309.

[13] Hunter, M.D., Mulligan, B.P., Dotta, B.T., Saroka, K.S., Lavallee, C.F., Koren, S.A. and Persinger, M.A. (2010) Cerebral dynamics and discrete energy changes in the personal physical environment during intuitive-like states and perceptions. Journal of Consciousness Exploration \& Research, 1, 1179-1197.

[14] Persinger, M.A. and Saroka, K.S. (2012) Protracted parahippocampal activity associated with Sean Harribance. International Journal of Yoga, 5, 140-145. doi:10.4103/0973-6131.98238

[15] Mulert, C., Jager, L., Schmitt, R., Bussfeld, P., Pogarell, O., Moller, H.J., Juckel, G. and Hegerl, U. (2004) Integration of fMRI and simultaneous EEG: Towards a comprehensive understanding of localization and time course 
of brain activity in target detection. NeuroImage, 22, 8394. doi:10.1016/j.neuroimage.2003.10.051

[16] Decoursey, T.E. (2003) Voltage-gated proton channels and other proton transfer pathways. Physiology Reviews, 83, 475-579.

[17] Lehmann, D., Grass, P. and Meier, B. (1995) Spontaneous conscious overt cognition states and brain electric spectral states in canonical correlations. International Journal of Psychophysiology, 19, 41-52. doi:10.1016/0167-8760(94)00072-M

[18] Tsang, E.W., Koren, S.A. and Persinger, M.A. (2004) Power increases within the gamma range over the frontal and occipital regions during acute exposures to cerebrally counterclockwise rotating magnetic fields with specific derivatives of change. International Journal of Neuroscience, 114, 1183-1193. doi:10.1080/00207450490475643

[19] Stark, T., Nissila, J., Aunio, A., Abou-Elseoud, A., Remes, J., Nikkinen, J., Timonen, M., Takala, T., Tervonen, O. and Kiviniemi, V. (2012) Stimulating brain tissue with bright light alters functional connectivity in brain at the resting state. World Journal of Neuroscience, 2, 81-90. doi:10.4236/wjns.2012.22012

[20] Rushworth, M.F., Behrens, T.E. and Johansen-Berg, H. (2006) Connection patterns distinguish 3 regions of human parietal cortex. Cerebral Cortex, 16, 1418-1430. doi:10.1093/cercor/bhj079

[21] Angel, A. and Klink, R. (1993) Differential responsiveness of stellate and pyramidal-like cells of the medial entorhinal cortex Layer II. Journal of Neurophysiology, 70, 128-143.

[22] Olivares, F.P. and Schuknencht, H.F. (1979) Width of the internal auditory canal in histological studies. Annals of Otologolgy, Rhinology and Larynegology, 198, 316-323.

[23] Persinger, M.A. (2010) 10 - 20 Joules as a neuromolecular quantum in medicinal chemistry: An alternative approach to myriad molecular pathways? Current Medicinal Chemistry, 17, 3094-3098. doi:10.2174/092986710791959701

[24] Sun, Y., Wang, C. and Dai, J. (2010) Biophotons as neural communication signals demonstrated by in situ biophoton autography. Photochemical and Photobiological Sciences, 9, 315-322. 